\title{
LOS NIÑOS QUEBRADOS DEL FRANQUISMO. LA VIVENCIA DE LA DISCAPACIDAD EN UN COLEGIO DE EDUCACION ESPECIAL DE LA ANIC*
}

\author{
The broken children of Francoism. The experience of disability \\ in a special education school run by the ANIC
}

\section{Salvador Cayuela Sánchez ${ }^{\alpha}$ y Mercedes Del Cura González ${ }^{\beta}$}

Fecha de recepción: 09/04/2020 • Fecha de aceptación: 04/06/2021

Resumen. Desde finales de los años sesenta, el régimen franquista potenció la creación de instituciones y centros destinados a la educación y formación de los niños y jóvenes con discapacidad. Estas entidades, a menudo dependientes de la iniciativa privada, hicieron crecer la débil estructura de servicios y prestaciones que el franquismo había organizado hasta entonces para hacer frente al problema de la discapacidad. En este trabajo se analiza el funcionamiento, y la labor educativa desarrollada en uno de estos centros durante los últimos años del franquismo y los primeros de la transición democrática: el Centro de Educación Especial "Nuestra Señora de la Fuensanta» de Churra (Murcia). Para ello, se ha combinado la documentación de archivo con metodologías de investigación cualitativa, en concreto 7 entrevistas en profundidad, semiestructuradas, realizadas tanto a antiguos alumnos como a miembros del equipo docente y directivo del centro en nuestro periodo de estudio. A través de los recuerdos y las experiencias personales de los entrevistados y entrevistadas, en el artículo se muestra cuál fue su vivencia de la discapacidad y qué efectos tuvo en su vida su paso por el centro.

\footnotetext{
* Este artículo ha sido elaborado en el marco del proyecto de investigación «El discurso acerca de la discapacidad en el tardofranquismo y la Transición y su influjo sobre el proceso de cambio sociocultural en torno a la normalidad corporal y mental» (HAR2015-64150-C2-2-P), financiado por el Ministerio de Economía y Competitividad del Gobierno de España/Fondos FEDER.

$\alpha$ Facultad de Filosofía, Departamento de Filosofía, Universidad de Murcia. Edificio Luis Vives, Campus de Espinardo, 30100 Espinardo-Murcia, España. scayuela@um.es (iD https://orcid.org/0000-0002-1982-3301

$\beta$ Facultad de Medicina de Albacete, Departamento de Ciencias Médicas - Instituto de Investigación en Discapacidades Neurológicas/Unidad Asociada del CSIC, Universidad de Castilla-La Mancha. C/Almansa 14, 02008 Albacete, España. Mercedes.DelCura@uclm.es (D) https://orcid.org/0000-0002-5972-7293
}

Cómo citar este artículo: Cayuela Sánchez, Salvador y Del Cura González, Mercedes. «Los niños quebrados del franquismo. La vivencia de la discapacidad en un colegio de educación especial de la ANIC». Historia y Memoria de la Educación 15 (2022): 229-258 
Palabras clave: Discapacidad física; Tardofranquismo; Transición democrática; Educación especial; ANIC.

Abstract. Beginning at the end of the 1960s, the Franco regime promoted the creation of institutions and centres for the education and training of children and young people with disabilities. These entities, often dependent on private initiative, supplemented the lacking structure of services and benefits that Francoism had organized until then to deal with the problem of disability. In this paper we analyze the operation and the educational work developed in one of these centres during the last years of Franco's regime and the first years of the democratic transition: the Special Education Center "Nuestra Señora de la Fuensanta" in Churra (Murcia). To this end, we have combined archival documentation with qualitative research methodologies, specifically 7 in-depth, semi-structured interviews, carried out both with former students and with members of the teaching and management team of the centre during our period of study. Through the memories and personal experiences of the interviewees, the article provides an account of their experience of disability and conveys the effects that the patients' passage through the centre had on their lives.

Keywords: Physical disability; Late Francoism; Spanish Democratic Transition; Special education; ANIC.

\section{INTRODUCCIÓN}

Desde finales de los años cincuenta y principios de los sesenta, el gobierno franquista iba a prestar una renovada atención a la discapacidad física, retomando estrategias previas y articulando otras nuevas. ${ }^{1}$ Todo ello no era independiente claro está, ni del contexto nacional, ni tampoco de las dinámicas internacionales cuya influencia era cada vez más notable y evidente en España. ${ }^{2}$ La discapacidad y su gobierno - como cualquier otro ámbito de la vida humana- no es independiente de los

\footnotetext{
1 Salvador Cayuela Sánchez y José Martínez-Pérez, «El dispositivo de la discapacidad en la España del tardofranquismo (1959-1975): una propuesta de análisis», Asclepio. Revista de Historia de la Medicina y de la Ciencia 70, no. 2 (2018): 232-244, https://doi.org/10.3989/asclepio.2018.16; y José Martínez-Pérez y Mercedes Del Cura González, «Bolstering the greatness of the homeland: productivity, disability and medicine in Franco's Spain (1938-1966)», Social History of Medicine 28, no. 4 (2015): 805-824, https://doi.org/10.1093/shm/hkv060.

${ }^{2}$ Como muestra de esa influencia en el ámbito sanitario y educativo véanse: Rosa Ballester Añón, «España y la Organización Mundial de la Salud. La cuestión española y la puesta en marcha de políticas y programas de salud pública (1948-1970)» en Salud, enfermedad y medicina en el franquismo, coords. María Isabel Porras; Lourdes Mariño y María Victoria Caballero (Madrid: Catarata, 2019), 43-56; y Lorenzo Delgado Gómez-Escalonilla, «International Organizations and educational Change
} 
discursos, estrategias y procesos que, en una sociedad y en un momento determinado, establecen relaciones de dependencia mutua y permanente interrelación.

En 1957 Frank J. Safford —miembro consejero de la Organización Mundial de la Salud (OMS) para niños físicamente disminuidos-y Kurt Jansson — jefe del Servicio de Rehabilitación de la Dirección de Asuntos Sociales de la Organización de Naciones Unidas (ONU)_, tras una visita orientada a evaluar las infraestructuras españolas destinadas a las personas con discapacidad, publicaban su influyente Programa Nacional de Rehabilitación de niños físicamente disminuidos, cuyo principal objetivo era «asesorar mejor al gobierno español, con vistas a poner en marcha, lo más rápidamente posible, un programa nacional de rehabilitación».3 Solo un año más tarde el gobierno franquista organizaba el primer Simposio Médico Social del Instituto Nacional de Previsión, dedicado a la «Rehabilitación del presunto inválido y Seguridad Social» ${ }^{4}$. Estos dos hechos son indicativos del creciente interés que tenía el régimen por este fenómeno y anticipan la orientación de las políticas de la discapacidad que impulsaría el gobierno tras la aprobación en 1959 del llamado Plan de Estabilización económica.

En este contexto, la implantación progresiva de la organización científica del trabajo — que ya antes había impulsado nuevas estrategias en el gobierno de la discapacidad física ${ }^{5}$ aparecía entonces como un requisito para la consolidación del crecimiento económico. También los «minusválidos ${ }^{6}$ debían amoldar sus cuerpos, sus actitudes y sus aptitu-

in Spain during the 1960s", Encounters in Theory and History of Education 21 (2020): 70-91, https:// doi.org/10.24908/encounters.v21i0.14334.

${ }^{3}$ Frank J. Safford, F. y Kurt Jansson, Programa Nacional de rehabilitación de niños físicamente disminuidos (Informe de una misión en España) (Madrid: Ministerio de la Gobernación-Dirección General de Sanidad, 1957). Sobre esta cuestión véase: Rosa Ballester Añón, «Los organismos sanitarios internacionales y la rehabilitación de los niños con discapacidades físicas (1948-1975)», Revista de Estudios do Seculo XX 12 (2012): 89-101, http://dx.doi.org/10.14195/1647-8622_12_5.

${ }^{4}$ VV. AA., I Symposium Médico-Social en el I.N.P. Tema de estudio: rehabilitación del presunto inválido y Seguridad Social (Madrid: Instituto Nacional de Previsión, 1959).

5 José Martínez-Pérez, «La Organización Científica del Trabajo y las estrategias médicas de seguridad laboral en España (1922-1936)», Dynamis. 14 (1994): 131-158.

6 A lo largo del periodo analizado en este trabajo, se usaron distintos calificativos para designar a las personas con discapacidad. La falta de acuerdo existente a nivel terminológico, que también afectó al ámbito administrativo, dio lugar a situaciones de desprotección y generó descontento en el colectivo. 
des a las exigencias del nuevo mercado laboral. ${ }^{7}$ Alejados de los tradicionales modelos de la beneficencia, "huyendo de soluciones caritativas y simplistas del sistema de subvenciones», ${ }^{8}$ las personas con discapacidad física debían pues formarse y adaptar sus cuerpos a las nuevas exigencias y posibilidades laborales del sistema económico neocapitalista. ${ }^{9}$

La vinculación con los Organismos Internacionales no solo iba a permitir al régimen satisfacer este objetivo, sino que también le iba a ayudar a ganar legitimidad a nivel internacional al ofrecer una imagen de estado "socialmente responsable». ${ }^{10}$ Siguiendo sus recomendaciones el gobierno franquista iba a impulsar la educación y formación profesional especializada destinada a las personas con discapacidad y, muy especialmente, a los niños. Según los expertos de la época, más allá del fundamental factor económico, esta formación es la que les iba a permitir disfrutar de una vida lo más completa posible, acorde siempre con sus posibilidades y limitaciones. ${ }^{11}$ Así, a lo largo de los años sesenta se impulsó la creación de centros educativos (sobre todo de gestión privada), se reguló la formación de profesorado especializado, y se pusieron en marcha becas destinadas a sufragar la formación de niños y jóvenes con discapacidad. ${ }^{12}$ Todas estas medidas, en el ámbito educativo, cristalizaron en la aprobación en 1970, de la Ley General de Educación, ${ }^{13}$ una

Véase: Gildas Bregàin, «Los debates públicos para sustituir el calificativo de inválido. (Argentina y España, de 1930 a 1970)», en Alter-habilitas: Percezione della disabilità nei popoli, ed. Silvia Carraro (Verona: Alteritas, 2018), 65-87.

7 Cayuela y Martínez-Pérez, «El dispositivo de la discapacidad», 236.

8 A. López Fernández y J. Bataller Sallé, «Importancia de la rehabilitación en los planes de la Seguridad Social», en VV. AA., I Symposium Médico-Social en el I.N.P. Tema de estudio: rehabilitación del presunto inválido y Seguridad Social (Madrid: Instituto Nacional de Previsión, 1959), 305-309.

9 José Martínez-Pérez, «Work, Disability and Social Control: Occupational medicine and Political Intervention in Franco's Spain (1938-1965)», Disability Studies Quarterly 37 no. 4 (2017), http://dx. doi.org/10.18061/dsq.v37i4.6098.

${ }^{10}$ David Brydan, Franco's Internationalist: social experts and Spain's search for legitimacy, (Oxford: Oxford University Press, 2019).

${ }^{11}$ José Bosch Marín et. al. (1958), Encuesta sobre niños físicamente disminuidos en España (datos provisionales) (Madrid: Ministerio de Gobernación, Dirección General de Sanidad, 1958), 63.

12 Mercedes Del Cura y Salvador Cayuela Sánchez, «De la caridad a los derechos: la discapacidad en el franquismo y la transición democrática», en El Estado del bienestar: entre el franquismo y la transición, eds. Damián González y Manuel Ortiz (Madrid: Silex, 2020), 259-288.

${ }_{13}$ Manuel de Puelles Benítez, Educación e ideología en la España contemporánea (Madrid: Tecnos, 1999), 319 y ss. 
norma con una clara convicción universalista que contemplaba la inclusión en el sistema educativo de aquellos niños afectados por deficiencias físicas, sensoriales e intelectuales que hasta entonces habían sido excluidos del sistema. A ello se refería explícitamente el artículo 12.2 de la ley, donde se afirmaba que «estarán también incluidas en el sistema educativo las modalidades que vengan exigidas por las peculiaridades de los alumnos, de los métodos y las materias». Según se apuntaba en el capítulo VII del título I, artículo 49.1, con esta reforma el gobierno aspiraba a "preparar, mediante el tratamiento educativo adecuado, a todos los deficientes e inadaptados para una incorporación a la vida social, tan plena como sea posible en cada caso, según sus condiciones y resultado del sistema educativo; y a un sistema de trabajo en todos los casos posibles, que les permita servirse a sí mismos y sentirse útiles a la sociedad». ${ }^{14}$ La ley equiparaba, por tanto, la educación especial a la ordinaria y garantizaba, al menos a nivel teórico, la formación de todos los niños con capacidades diferentes, disponiendo su escolarización en centros especiales (cuando las deficiencias que presentaban eran profundas) o en unidades especiales organizadas dentro de los centros ordinarios (art. 51).

Uno de los organismos que más decididamente impulsó la creación de centros de educación especial en nuestro país fue la Asociación Nacional de Inválidos Civiles (ANIC). Creada en 1958 y definitivamente constituida en 1961, la ANIC articuló una concepción compleja de la discapacidad física que comprendía desde la educación escolar básica, hasta espacios de rehabilitación y servicios de ortopedia, y centros de formación profesional para los más mayores. En este trabajo analizaremos precisamente el funcionamiento, la estructura y la labor educativa de uno de estos colegios: el Centro de Educación Especial «Nuestra Señora de la Fuensanta» de Churra (Murcia). Para ello, combinaremos la investigación de archivo y la reflexión crítica, con metodologías de investigación cualitativa, en concreto 7 entrevistas en profundidad, semiestructuradas, concedidas entre enero de 2018 y junio de 2020, y realizadas concretamente a dos miembros del equipo docente y directivo, a cuatro antiguas alumnas y a un antiguo alumno del colegio. A estos entrevistados y entrevistadas nos referiremos de forma anónima utilizando

${ }^{14}$ Ley 14/1970 de 4 de agosto. Boletín Oficial del Estado de 6 de agosto de 1970, n. ${ }^{\circ} 187$. 
el acrónimo ED (Educación de la Discapacidad) seguido de un número (por ejemplo, ED_1). En este punto, y siempre admitiendo la inherente carga subjetiva del recuerdo personal, ${ }^{15}$ así como el limitado número de testimonios recogidos, consideramos que estos relatos constituyen una fuente de información inestimable para una mejor comprensión de los desarrollos acaecidos en relación con la educación de las personas con discapacidad en nuestro periodo y contextos de análisis, así como de la vivencia de la discapacidad.

\section{EL CENTRO DE EDUCACIÓN ESPECIAL «NUESTRA SEÑORA DE LA FUENSANTA» DE CHURRA (MURCIA)}

Antes de comenzar con nuestro análisis, nos parece conveniente trazar brevemente la historia de la Asociación Nacional de Inválidos Civiles (ANIC), institución que, tal como hemos señalado, se encargaría de la puesta en marcha del colegio que aquí nos sirve como caso de estudio. ${ }^{16}$ Los inicios de esta "asociación paraestatal» debemos buscarlos en los años cuarenta, cuando algunos miembros de las asociaciones de inválidos, conscientes de la dificultad de financiar sus organizaciones, se plantearon la posibilidad de crear una organización similar a la ONCE que, en ese momento, gozaba de independencia económica gracias a la explotación del cupón de lotería. ${ }^{17}$ En 1947, una comisión integrada por personas con discapacidad física y representantes de la ONCE elaboraba

\footnotetext{
15 Albrecht Lehmann, «Cultural Anthropology and Narratology», en Anthropological Perspectives. Tools for the Analysis of European Societies/Perspectivas antropológicas. Materiales para el análisis de las sociedades europeas, eds. Klaus Schriewer y Salvador Cayuela Sánchez (Murcia/Münster: Waxmann/Editum), 69-91. Y Klaus Schriewer y Manuel Nicolás Meseguer, «El relato de justificación. Una herramienta para el análisis del franquismo», Revista Murciana de Antropología, 23 (2016): 85102, https://revistas.um.es/rmu/article/view/273781.
}

${ }^{16}$ Sobre el origen y desarrollo de la asociación véanse: Gildas Brégain, «'Nous ne demandons pas la charité. Nous voulons du travail!'». La politique franquiste d'assistance aux invalides», Alter. European Journal of Disability Reserach, 7 (2013): 206-221, https://doi.org/10.1016/j.alter.2013.04.003. Emilia Martos, Personas mayores y diversidad funcional física e intelectual durante la transición a la democracia. Problemática, reivindicación y actuación en el ámbito nacional y local, el ejemplo de la provincia de Almería (Almería: Universidad de Almería, Tesis Doctoral, 2014), 269-279. Y Emilia Martos, «Trabajo y "minusvalía” durante el primer Franquismo: La asociación nacional de inválidos civiles", en La Historia: lost in translation?, eds. Damián A. Gonzalez y Manuel Ortiz Pérez (Albacete: Ediciones de la Universidad de Castilla-La Mancha, 2017), 2787-2796.

${ }^{17} \mathrm{El}$ hecho de que la ONCE detentara el monopolio del cupón provocó enfrentamientos con otros colectivos de personas con discapacidad, ya que la financiación de sus asociaciones dependía básicamente de las cuotas de los socios, de donaciones, de rifas, de la venta de la lotería y de magras subvenciones estatales. La ONCE nunca renunció a la exclusividad, pero aceptó incorporar entre sus 
un plan para la creación de un organismo de Inválidos Civiles, que sería elevado a la Dirección General de Beneficencia. Dicho plan se basaba en la unión de todas las asociaciones provinciales en una única organización nacional, la única estrategia posible para solventar el gran problema de la carencia de iniciativas laborales, educativas o de inserción de las personas portadoras de deficiencias físicas en España. No obstante, el gobierno no se mostró dispuesto a ocuparse de esta cuestión hasta mediados de los años cincuenta, cuando en el III Congreso Nacional de Trabajadores de 1956 se estipuló que la Organización Nacional de Inválidos quedase encuadrada en la Organización Sindical.

Lejos de certificarse entonces la creación de la futura ANIC, este aparente impulso suscitó recelos en el Ministerio de Gobernación, que pasó a competir con el de Trabajo — del que dependía la Organización Sindical- por el control de la nueva asociación. No se trataba de una cuestión menor en absoluto, pues la adscripción de la futura ANIC a uno u otro ministerio podía marcar radicalmente su destino: depender de la Organización Sindical, de marcado carácter falangista, supondría que la nueva asociación se encuadrara en el ámbito laboral, inspirada de una determinada concepción de la unión social y la solidaridad comunitaria obligatoria. Por otro lado, si quedaba vinculada al Ministerio de la Gobernación, dominado ya por los llamados tecnócratas cercanos al Opus Dei, supondría situarla dentro de las estrategias de la beneficencia, emparentada con el concepto religioso de caridad que promulgaban sus dirigentes. ${ }^{18}$

Como iba a suceder en tantos otros ámbitos en aquellos años, Gobernación terminaría rigiendo los designios de la nueva asociación, que era finalmente creada por Orden de 29 de noviembre de $1958 .{ }^{19}$ La nueva agrupación nacional —que eliminaba la posibilidad de formación de nuevas asociaciones de inválidos civiles y aglutinaba las ya existentes-, tenía la consideración de entidad benéfico-particular y quedaba

vendedores a personas con discapacidad física. Véase: Martos, «Trabajo y "minusvalía” durante el primer franquismo», 2791.

18 Gildas Brégain, «'Nous ne demandons pas la charité. Nous voulons du travail!'», 214-216. Y Emilia Martos, Personas mayores y diversidad funcional, 272.

19 Boletín Oficial del Estado de 22 de diciembre de 1958, n. ${ }^{\circ}$ 305. Los estatutos de la Asociación se ampliarían un año más tarde a través de la Orden de 14 de diciembre de 1959. Boletín Oficial del Estado de 24 de diciembre de 1959, n. ${ }^{\circ} 307$. 
organizada por unas Juntas (nacional y provinciales) y sus respectivas comisiones delegadas. Su presidente nacional era designado por el Ministerio de Gobernación, y su designación se hacía a propuesta de la Dirección General de Beneficencia y Obras Sociales, y previo informe del Patronato de Rehabilitación y Reeducación de Inválidos. La asociación estaba abierta a todas las personas con algún tipo de deficiencia física o funcional que les impidiese o dificultase el desarrollo de los quehaceres de la vida normal, ya fuese el origen de dichas carencias accidentes de trabajo o tráfico, malformaciones congénitas o derivadas de enfermedades — como el caso de la polio_-, o resultado de lesiones de guerra. ${ }^{20}$

Los objetivos principales de la nueva organización eran por lo demás variados y complejos: informar a las autoridades de la situación de las personas con discapacidad física; plantear las posibles soluciones para dichos problemas; o coordinar los esfuerzos de las instituciones estatales y privadas involucradas en el cuidado y la atención a las personas con discapacidad física y potenciar la integración laboral y social de sus afiliados. ${ }^{21}$ No obstante, la ANIC no consiguió influir sensiblemente en la empleabilidad de las personas con discapacidad física, su principal objetivo, y tampoco poner en marcha un proyecto parecido por ejemplo al cupón de la ONCE. En este sentido, la asociación no pudo ir más allá de la reserva de la explotación del servicio de guardacoches concertado con algunos municipios, la administración de quioscos, la venta de entradas en espectáculos y de billetes fraccionados de la lotería nacional, por lo que su capacidad para "colocar» a sus miembros fue muy limitada. ${ }^{22}$ Con todo, ya a mediados de los años sesenta, la ANIC iba a ser capaz de impulsar la creación de dos centros de formación profesional, uno en

\footnotetext{
${ }^{20}$ La ANIC también surgía con el objetivo de encauzar las peticiones de los mutilados de guerra republicanos que habían sido excluidos del Cuerpo de Mutilados de Guerra creado por el franquismo antes de acabar la guerra civil.

${ }^{21}$ Fernando Tamés Seminario, «La Asociación Nacional de Inválidos. Sus problemas, sus inquietudes, sus aspiraciones». En Charlas Radiofónicas. El problema de los disminuidos físicos y su rehabilitación. (Madrid: Patronato Nacional de Rehabilitación de Inválidos, 1963), 41-48. Tamés, abogado de origen vasco y con discapacidad motora, había sido profesor adjunto en la Cátedra de derecho procesal de la Universidad de Madrid durante los años cuarenta. Su experiencia en al ámbito jurídico y su discapacidad lo convirtieron en el candidato perfecto para llevar las riendas de la asociación y ser su imagen pública desde su nombramiento, en el 59, hasta su muerte en 1972.

22 J. S. Canales, «La ANIC, al cumplir diez años de labor en el sector de los minusválidos españoles, presenta un alentador balance de realizaciones», $A B C$ (Sevilla), 18 de marzo de 1972. La asociación creó además centros de empleo protegido en Madrid, Albacete, Barcelona, Burgos, Cuenca, Granada, Jaén y Zaragoza.
} 
Madrid —dedicado a la enseñanza técnica del montaje de radio, televisores y electricidad - y otro en Toledo — de damasquinado y grabado en metal-, a los que se unirían poco después otros dos en Albacete y La Alberca. ${ }^{23}$ En esta línea, la asociación llegó a ofrecer cursos de electricidad del automóvil, confección de calzado ortopédico, montadores y reparadores de electrodomésticos, costureras y tejedoras de alfombras de artesanía, etc. ${ }^{24}$

La precaria situación financiera de la ANIC en sus primeros años de existencia —dependiente esencialmente de las ayudas de la ONCE, cuestaciones ocasionales y las propias cuotas de los socios- le impidió desarrollar plenamente sus estrategias iniciales, pero su situación económica mejoró sensiblemente desde 1968 al ser incluida como beneficiaria en el II y el III Planes de Desarrollo (1968-1971 y 1971-1974).25 Aunque las necesidades del colectivo de personas con discapacidad excedían con mucho las posibilidades abiertas con la nueva financiación, su carácter regular le iba a permitir afrontar proyectos más ambiciosos y de largo alcance, entre los cuales cabe destacar la creación de varios centros escolares de educación especial y de referencia nacional construidos desde finales de los años sesenta en Barcelona, Albacete, Jaén y Murcia. ${ }^{26}$ Estos centros sin duda suponían un avance considerable en las políticas educativas de atención a la discapacidad en España, cristalizando tanto los proyectos impulsados por la propia ANIC en años anteriores, como las estrategias educativas diseñadas entonces por los especialistas en Educación Especial. ${ }^{27}$

En este contexto se aprobaría por Orden de 9 de marzo de 1971 la creación del Centro de Asistencia y Educación Especial de ANIC «Nuestra Señora de la Fuensanta» en la pedanía murciana de Churra. ${ }^{28}$ El centro

\footnotetext{
${ }^{23}$ Brégain, «'Nous ne demandons pas la charité. Nous voulons du travail !'», 216.

24 Martos, Personas mayores y diversidad funcional, 275.

25 Brégain, «'Nous ne demandons pas la charité»", 219.

26 «La ANIC estructura grandes centros de enseñanza. En pro de la rehabilitación de los minusfísicos». A.N.I.C. Boletín Informativo. Delegación de Zaragoza 7, n. 70 (1971), s.p.

27 VV. AA. «Monográfico Educación Especial. Problemas y técnicas», Bordón. Revista de Orientación Pedagógica, 164-165 (1969).

28 Boletín Oficial del Estado de 12 de abril de 1971. Recogido en: Solicitud de Fernando Tamés Seminario, presidente de la ANIC al Ministerio de Educación y Ciencia, 6 de noviembre de 1972.
} 
iba a contar con ocho unidades escolares — cuatro de niños y cuatro de niñas-, con plazas gratuitas, y con las «instalaciones Gimnástico-Deportivas necesarias para la rehabilitación física de los escolares». El coste total de las obras e instalaciones, realizadas entre octubre de 1968 y junio de 1971, ascendió a algo más de 27 millones y medio de pesetas y fue subvencionado por la Dirección General de Política Interior y Asistencia Social, mientras que el Ministerio de Educación y Ciencia se ocupó de la dotación de mobiliario y material. ${ }^{29}$ El centro se abrió en el curso escolar 1971-1972, y en el curso siguiente ya contaba con un total de 194 alumnos y alumnas - 115 en régimen de internado-, realizando los estudios de $1 .^{\circ}$ a $8 .^{\circ}$ de Educación General Básica (EGB). De hecho, el elevado número de alumnos supuso el desdoble de $6 .^{\circ}$ de EGB en dos grupos ya en el curso 1973-1974.

Como ponía de relieve el primer informe elaborado por la Inspección Provincial de Enseñanza Primaria de Murcia, el colegio estaba llamado a ser un referente en la educación de niños con discapacidad física en España. En dicho informe se señalaba que:

Estimadas las especiales circunstancias concurrentes en la referida Instrucción que, tanto en el orden material como en el pedagógico (los que cabe juzgar a esta inspección) son magníficos y eficientes, con la lógica aspiración de llegar a calificarse de modélicos en su género [...] Considerando la singular utilidad que despliega el Centro, permitiendo una reincorporación de niños en edad escolar a la vida ordinaria, en orden a la cultura y a su actividad psicofísica con apenas disminución solamente a lo que concierne a sus limitaciones motóricas. ${ }^{30}$

Todo ello justificaba su consideración como «Centro de Educación General Básica, de Educación Especial, con residencia», y la consiguiente capacidad de conceder los certificados de EGB — solicitados al Ministerio- para sus alumnos, así como optar a las subvenciones que permitieran «disponer de una modernísima y espléndida instalación, llena de

\footnotetext{
${ }^{29}$ Informe de Instancia de Clasificación y Transformación de Centros de Enseñanza de la ANIC del 30 de junio de 1972 (Expediente INSERSO, 44066/36), Archivo General de la Región de Murcia.

30 Informe de 17 de julio de 1972 de la Inspección Provincial de Enseñanza Primaria de Murcia (Expediente INSERSO, 44066/36), Archivo General de la Región de Murcia.
} 
alma y contenido». No obstante, y como Miguel Pereyra Etcheverría ${ }^{31}$ -el primer director del colegio de Churra- nos comentó, la realidad del centro al menos en aquellos primeros años era bien distinta:

[Yo tenía relación con la ANIC por mi accidente] y escuché que buscaban a un director de un colegio de discapacitados en Murcia [y] lo solicité. Entonces a principios de septiembre del 71 viajé a Murcia [desde Madrid] y me encontré un colegio en Churra ya construido con las ayudas del Plan de Desarrollo. Un colegio inacabado y sin mobiliario [...] hubo que rediseñar, acabar las obras y amueblar. [Pero] no había dinero. [Y] pensé: si ANIC depende de Gobernación, lo que empecemos a tocar con los proveedores pues se terminará por pagar. La Administración siempre paga. Entonces como pudimos preparamos el colegio, y a mitad de noviembre ya pudieron venir los primeros 100 alumnos. Físicos, la mayoría eran secuelas de polio, de toda España. Por ejemplo, de Toledo teníamos 8, del País Vasco 15... [No puedes imaginar] lo que aprendí al poner en marcha ese colegio. Vivimos así dos años, sin presupuesto.

Nacido en San Sebastián en 1938 - y con una paraplejía desde 1966 como consecuencia de las lesiones sufridas en un accidente de tráfico-, Miguel Pereyra es sin duda uno de los nombres propios en el mundo de la discapacidad física en la España de los últimos cincuenta años, y como vemos una persona clave en la historia del colegio de la ANIC en Churra. De hecho, parece que fue la gran inventiva de su primer director lo que salvó en más de una ocasión la precaria situación económica con la que nació el colegio, llamado a ser —no lo olvidemos- de «referencia nacional» en la atención a los niños con discapacidad física. Así lo podemos intuir a partir del siguiente fragmento de entrevista:

Cuando llegó el segundo año [todavía sin presupuesto], llamé al Administrador del colegio y le dije: «Mira, nos quedan 3 o 4 meses para el fin de curso. Compra todos los alimentos que necesitemos ahora, porque no tenemos dinero para pagar». Y cuando vuelve me dice que el empresario que nos suministra no nos da

\footnotetext{
${ }^{31}$ La referencia explícita a Miguel Pereyra Etcheverría -a quién correspondería el código ED_1cuenta claro está con el expreso consentimiento de nuestro entrevistado, pero se debe especialmente a que sería prácticamente imposible mantener aquí su anonimato, debido a su destacado papel en el mundo de la discapacidad en la España de los últimos cincuenta años.
} 
nada más, porque no cobra. ¿Y entonces yo qué hago? Pues le digo al administrador: "¿Dices que tiene tres almacenes verdad? Pues vete a otro almacén como si tú no supieras nada, porque a lo mejor no ha dado todavía la orden». Y entonces fue a otro almacén, y llegó más tarde con la furgoneta cargada hasta arriba.

Esta situación de penuria económica se dejó sentir incluso entre los niños y niñas allí internos, como podemos observar en el siguiente verbatim — que nos consta no sucedió con Miguel Pereyra como director-: «Miguel Pereyra era una excelente persona, pero lo echaron del colegio [...] Yo he llegado a pasar hambre allí. Y fue cuando mi padre dijo: "Se ha terminado". Y me sacó de hecho a mitad de curso. Pasando hambre, nos trataban mal» (ED_2). Aunque ningún otro de nuestros entrevistados o entrevistadas nos ha contado situaciones tan extremas, lo cierto es que han sido muy frecuentes en nuestras conversaciones las referencias al rancho típicamente carcelario y restringido del colegio, así como el "olor a sopa» de los pasillos y las «salchichas de lata» como un auténtico lugar común en sus recuerdos:

Yo con las comidas recuerdo que muy mal. [De hecho] hace poco lo hablaba con una compañera del País Vasco, que me decía que no había podido comer más sopas [desde que salió], y tampoco salchichas. Claro, aquellas comidas que te las tenías que comer sin más... Es que era el mismo olor, coincidimos en cosas que... De hecho, yo nada, me acuerdo que no comía. En el comedor, como siempre había chuscos de pan, le echábamos un poco de aceite, un poco de sal y... ¡qué rico! (ED_5).

Es curioso en este sentido como ese supuestamente escaso y monótono rancho no era percibido del mismo modo por —al menos algunosde los niños allí internos:

[El colegio] muy bien. Lo único la comida. Cuando yo entré, nos tenían un poco más escasos. En la mesa que nosotros estábamos con un compañero, Pepe de Molina, cuando había habichuelas a la gente no le gustaban, y entre los dos nos comíamos todas las habichuelas, o todas las lentejas... Pero yo creo que bien. Se repetían un poco las cosas, pero nos trataban bastante bien, por lo que yo recuerdo (ED_6). 
Por lo demás, Miguel Pereyra es recordado de hecho por todos nuestros entrevistados y entrevistadas con un profundo sentimiento de cariño y admiración, garante de esa «otra forma de pensar la discapacidad» que comenzó a poner en práctica en el centro de Churra. Pero también él tuvo que aprender entonces cómo gobernar aquel colegio, con circunstancias y particularidades que excedían — claro está- lo puramente económico. En este sentido, por ejemplo, es sumamente interesante recordar cómo se llegaron a introducir en Churra técnicas pedagógicas innovadoras en educación especial —que comprendían desde la instrucción de los escolares hasta la rehabilitación y ortopedia- orientadas a la atención personal, atentas a las circunstancias individuales de cada escolar y preocupadas por la formación profesional y la posterior inserción laboral del alumnado. Estas nuevas aproximaciones fueron precisamente importadas desde un centro hermano de la ANIC en Barcelona, creado ya en 1967. Así lo recordaba el propio Pereyra:

En ese segundo año [1972] conocí a la directora de Barcelona que era psicóloga. Del colegio de la ANIC, que tenían 150 niños, de los cuales 50 iban en silla de ruedas. Yo oía hablar de la señorita Cabellos, y yo pensaba que la señorita Cabellos era la funcionaria del Ministerio de Educación. Pero un día voy a Madrid y me dice el presidente [de la ANIC]: «Tengo aquí a la directora de Barcelona con todo el equipo de maestros que viene a verme, ¿quieres estar en la reunión?». Y dije que encantado. Nos tiramos de 10 de la mañana hasta las 3 y media de la tarde. Yo no conocía el colegio de Barcelona, y claro, me resultó interesante. Y resulta que a mí me odiaban en Barcelona [...] porque cuando pedían algo les decían que el director de Murcia decía que no les hacía falta. Y entonces, al salir de la reunión le dije a Mari Carmen [Cabellos] que me resultaba interesante seguir hablando con ella y estar en contacto. Le pregunté si era accesible su hotel [en Madrid], y si no pues que se viniera a casa [de mis padres]. Y vino, y quedamos en que visitaríamos juntos las escuelas [de Murcia y Barcelona]. Y al cabo de un mes o así me llama que viene a Murcia a ver las aulas y me puso a parir: «Le falta mano femenina, iperece esto un cuartel y hay niños de 6 años!». Y me dijo: «Bueno, ¿por qué no vienes a ver la escuela de Barcelona?». Y en menos de un mes fui a Barcelona y realmente era estupenda. Ellos estaban 
haciendo cursos de pedagogía terapéutica para los físicos, conocían muy bien el tema y de hecho fueron los primeros que hablaron de integración. Nos decían que la escuela tiene que ser niveladora, y después tienen que ir al instituto, no pueden ir a centros especiales. Porque entonces, el que era especial [tenía una discapacidad física] pues lo consideraban deficiente mental.

Tan solo unas semanas después Miguel Pereyra solicitó permiso al delegado de Educación y Ciencia en Murcia para visitar el colegio de Barcelona con todos los educadores y profesores del centro de Churra. ${ }^{32}$ Ya en Barcelona, además, los visitantes seguirían un curso intensivo de «Educación Especial de deficientes motóricos», organizado expresamente por el Instituto de Ciencias de la Educación de la Universidad de Barcelona. El curso, organizado en colaboración con la Escuela Normal y de la Inspección Técnica, se celebró entre el 2 y el 26 de enero de 1973, y fue convalidado por el Ministerio de Educación, lo que les valdría la concesión de una titulación oficial. La realización de este curso conllevaba de hecho la pérdida de 20 días de clase en el mes de enero, sustituidos mediante la supresión de prácticamente todos los días de vacaciones de Semana Santa tanto para trabajadores como para los niños allí inscritos. Todo ello nos permite señalar dos aspectos de capital importancia: por un lado, la destacable implicación tanto de la dirección del nuevo colegio como de su profesorado en la mejora de las técnicas educativas en beneficio de los alumnos; y, por otro lado, la relativamente escasa preparación especializada previa con la que contaban. No obstante, parece que aquella formación tuvo un impacto muy positivo en la educación de los niños que estudiaban en el colegio, como podemos concluir de varios fragmentos de entrevista. A ello se refería esta entrevistada: «A mí me parece que [la formación estaba] muy bien, y además muy exigente. No te dejaban nunca. Y si estabas castigado, tenías que cumplir todo el castigo. En general muy bien» (ED_5). O esta otra:

Hubo muy buenos profesores. Gente joven que acababa de sacar la carrera de profesor. De hecho, los mayores teníamos 16 años y ellos veintipocos en aquel momento. [Piensa además que] en aquellos años solo había «la maestra del pueblo», que lo daba

\footnotetext{
32 Informe del Centro de Educación Especial de ANIC en Churra de 18 de diciembre de 1972, n. ${ }^{\circ}$ 2936 (Expediente INSERSO, 44066/36), Archivo General de la Región de Murcia.
} 
todo, la misma persona. Y ahí era mucho más adelantado. Lo que hay hoy en día, allí ya existía entonces. Gente muy preparada. Los psicólogos nos ayudaron muchísimo para enfrentar la vida, lo que sería nuestro futuro. Que si no estudiábamos seríamos guardacoches. Todo eso a mí me hizo mucha mella y la preparación me vino muy bien. De hecho, cuando salí de ahí estudié música, me apunté al conservatorio, hice Administración... (ED_3).

Pero además de estos estudios de EGB, el centro contaba con talleres de formación profesional adaptados a las capacidades de los internos. Así recordaba uno de nuestros entrevistados —afectado de espina bífida- aquellos talleres de carpintería y albañilería, espacios donde no solo aprendía con «Don Manuel» ciertas destrezas manuales, sino que además le servían de refugio personal:

Yo estaba más centrado en unos talleres que teníamos ahí, que les decíamos «la granja», de cosas de carpintería y todo eso, y yo me iba de un sitio para otro. Yo con la gente no estaba muy relacionado, porque en aquella época era muy tímido, muy independiente, y yo me iba para el taller de albañil con Don Manuel que era el profesor. Porque yo ni jugaba fútbol ni nada [En el taller] teníamos martillos, cepillos, teníamos de todo. Porque Don Manuel era carpintero [...] Yo creo que trabajaba bastante, hacía muchos trabajos (ED_6).

A pesar de la buena gestión, aun salvando la escasez de recursos —al menos en sus primeros años-, del enorme impulso y la mejora de las técnicas pedagógicas que propició su inquietud personal y profesional, y del patente cariño que los niños le profesaban, Miguel Pereyra fue cesado como director antes incluso de terminar el curso 1974-1975. Así lo recordaba el propio Pereyra, acontecimiento tremendamente esclarecedor para comprender tanto el futuro de la ANIC como el de sus proyectos y centros educativos:

Estuve en el colegio tres cursos, el último incompleto porque en este tránsito murió el presidente de la ANIC, que era un tío soberbio, Fernando Tamés. Nosotros dependíamos de la ANIC, pero a través del Ministerio de Gobernación, donde entonces estaba el Camulo [Carlos Arias Navarro]. Desde algunos meses 
antes Mari Carmen [Cabellos, la directora del colegio de Barcelona con la que de hecho terminaría casándose] y yo decidimos que se acabó el que nos tomaran el pelo, que el director de Murcia y la directora de Barcelona nos pondríamos de acuerdo en lo que íbamos a pedir, y que plantearíamos la misma formulación. Al principio [tras la muerte de Fernando Tamés] el subdirector de Gobernación de Servicios Sociales me preguntó si yo estaría dispuesto a ser el presidente de la ANIC, y le dije que no, porque seguramente tendría que barrer muchas cosas, y a mí no me gusta barrer, sino construir [...] Y entonces nombran presidente a la persona menos discapacitada que he conocido nunca, que le faltaban solo dos dedos de la mano, era abogado. [Y poco después] recibo una llamada telefónica a las 12:30 o 1, y me dicen que ha ido para Murcia el nuevo presidente de la ANIC, que venía a pedirme la dimisión o a darme el cese. «Te lo aviso para que estés preparado». Y yo entonces llamé a Gobernación, al subdirector, y le dije lo que me acababan de comunicar, y si tenía el respaldo de la subdirección. Y me respondió: «Hombre, Miguel, acabamos de nombrarle, sería muy...». Y le respondí: «Bueno, pues ya me has contestado, sé que no tengo el respaldo». Entonces comí y me acosté, con premeditación y alevosía. Y nada más acostarme me avisan que había venido. Le hice esperar tres cuartos de hora. Y trae un carpetón y me dice que venía a pedirme mi dimisión o cese. Me dijo: «Toda esta carpeta son agravios contra ti». Y yo le dije: «Ni dimisión ni cese, ábreme un expediente, dime los cargos que tengo, y si no soy capaz de defenderme contra los cargos, césame». Al final me cesó.

Ya fuera por las crecientes demandas coordinadas desde los colegios de Murcia y Barcelona, por «eliminar» a quien en algún momento se había postulado como presidente de la ANIC, o simplemente como resultado de un cambio ministerial en las convulsas postrimerías del régimen franquista, aquel cese provocó al parecer una profunda consternación tanto entre los profesionales del centro como entre los propios alumnos. Así recordaba uno de los administrativos del colegio en aquellos años la marcha de Pereyra, y la relación con el nuevo presidente de la ANIC:

[Cuando] Miguel se va nos quedamos desamparados. Todos los que éramos [...] del grupo de trabajo de Miguel nos apuntan 
[con el dedo] y empiezan a echar gente a la calle. A mí no sé por qué no me echan, porque encabezo los escritos, llamo a Madrid... Una vez me llamó el presidente de ANIC y me dijo: «¿Sabe usted cuál es su trabajo en el colegio?». Y le respondí: «Sí». Y me dijo: «Pues dedíquese usted a trabajar. Porque cualquier día me levanto con el pie izquierdo y va usted a la calle». Digo: «Bueno, pues me parece muy bien. Le recuerdo que usted no es la máxima autoridad en este país. Hay más gente con la que se puede hablar. Y yo puedo hablar». No me salió mal [porque no me echaron]. Cuando el tío venía al colegio ponía las sirenas. Nos ponía a todos firmes [en el patio] y pasaba saludando como Franco. Y a mí no me daba la mano nunca, me saltaba (ED_7).

En lo que respecta a la situación económica del centro, tampoco parece que hubiera mejorado demasiado desde su apertura, como indica el hecho de que tanto Miguel Pereyra como María Carmen Cabellos solicitaran al recién constituido Servicio Social de Recuperación y Rehabilitación de Minusválidos Físicos y Psíquicos (SEREM) ${ }^{33}$, que se hiciera cargo de los colegios tutelados por la ANIC. Así lo recordaba Pereyra: «Le habíamos pedido al SEREM que se hicieran cargo del mantenimiento de las escuelas, porque la ANIC no era capaz. Eso ya se había hablado con el anterior [presidente de la ANIC], y estaba de acuerdo. Teníamos unas deudas inmensas».

Sea como fuere, el 23 de julio de 1978 el por entonces Centro de EGB para Minusválidos Físicos «Nuestra Señora de la Fuensanta» de Churra pasó a depender del SEREM, ${ }^{34}$ quién "mantuvo su destino» —en expresión de la documentación del Ministerio de Sanidad y Seguridad Social consultada ${ }^{35}$ hasta el año 1979. Ese mismo año, en concreto el 19 de octubre, un informe técnico ordenó el desalojo del colegio debido a anomalías surgidas en la edificación por movimientos en su estructura y cimentación, sobrevenidas por la existencia de «arcillas expansivas

\footnotetext{
${ }_{33}$ Mercedes del Cura González, «El Servicio Social de Recuperación y Rehabilitación de Minusválidos: de la valoración de capacidades a la atención integral (1970-1979)», en Salud, enfermedad y medicina en el franquismo, coords. María Isabel Porras; Lourdes Mariño y María Victoria Caballero (Madrid: Catarata, 2019), 262-280.

${ }^{34}$ Real Decreto 1724/78 de 23 de julio. Boletín Oficial del Estado de 20 de julio de 1978, nº 172.

${ }_{35}$ Informe del Ministerio de Sanidad y Seguridad Social de 30 de julio de 1981, n. ${ }^{\circ} 20389$ (Expediente INSERSO, 44066/36), Archivo General de la Región de Murcia.
} 
en el suelo».36 Tras su cierre, los alumnos fueron distribuidos por distintos centros de Murcia, y la mayor parte reubicados en el Centro de Recuperación de Minusválidos Físicos de Albacete (abierto en el curso 1972-1973). De hecho, aún en 1981 el Instituto Nacional de Servicios Sociales (INSERSO) — del que dependía el SEREM - ponía a disposición del Instituto Nacional de Educación el edificio que había albergado al antiguo colegio de la ANIC. A aquella propuesta el Ministerio de Educación respondería que la localidad de Churra ya contaba con puestos escolares suficientes, y que además la capacidad del centro era muy limitada, por lo que «estimaba que no procede la compra de dicho edificio».37 Uno de los centros de referencia nacional impulsados por la ANIC para la educación de niños portadores de deficiencias físicas llegaba así a su final, menos de diez años después de su apertura.

\section{EL COLEGIO DE CHURRA EN LA MEMORIA DE SUS ALUMNOS}

La historia del colegio es sobre todo la historia de los alumnos y alumnas que pasaron por sus aulas, y por ese motivo nos ha parecido relevante conocer cómo vivieron - y recuerdan hoy-su estancia en aquel colegio los niños y niñas allí educados. Después de todo, la apertura de un colegio de educación especial se presentaba como una oportunidad inestimable tanto para los escolares como para sus familias, un centro donde en principio podrían recibir una educación adecuada a sus necesidades, y donde además el «estigma de la discapacidad» —aún muy presente en el «medio normal»—38 quedaría mitigado. Así se refería a ello un antiguo alumno del colegio con el que tuvimos ocasión de conversar, comparándolo con su anterior experiencia en «colegios normales»: «En colegios normales estaba muy limitado y cuando me fui al colegio de Churra, pues era el rey. Porque podía más o menos correr un poco, entonces pues estaba bien» (ED_6).

\footnotetext{
${ }^{36}$ Informe del Ministerio de Sanidad y Seguridad Social de 19 de octubre de 1979, n. ${ }^{\circ} 12022$ (Expediente INSERSO, 44066/36), Archivo General de la Región de Murcia.

${ }^{37}$ Informe del Ministerio de Educación de 21 de septiembre de 1981, n. 06614 (Expediente INSERSO, 44066/36), Archivo General de la Región de Murcia.

38 Pamela K. Richardson, «The School as Social Context: Social Interaction Patterns of Children with Physical Disabilities», The American Journal of Occupational Theory, 56 (3) (2002): 296-304, https://doi.org/10.5014/ajot.56.3.296.
} 
No cabe duda, por lo demás, de que el hecho de que las plazas fueran gratuitas podía suponer un alivio económico para las familias, sujetas por otro lado a una presión económica importante derivada del coste tanto de las intervenciones quirúrgicas como de los aparatos ortopédicos requeridos por sus hijos e hijas. Así lo expresaba una de las antiguas alumnas del colegio con la que hemos tenido ocasión de conversar:

Yo fui al colegio del pueblo donde vivía, en una pedanía de Murcia. Pero luego llegó una carta sobre un colegio que habían abierto exclusivamente para personas con discapacidad. Éramos casi todos de polio, y nos arrumbaron ahí. Yo que era una cría con 7 años, en tercero de EGB, quería ir, pero mi padre no, porque era interna, y él no quería eso. Cuando llegó aquella carta [yo dije que si] porque en ese momento en el colegio te miran raro, y entonces yo dije que sí, que quería ir... Y finalmente fui (ED_2).

Otros padres se mostraron bastante más entusiastas con la idea, convencidos de la necesidad de impulsar la autonomía de sus hijos e hijas con discapacidad en un medio alejado del ambiente muchas veces sobreprotector de sus familias:

Mi padre veía una protección tan grande [en casa] que se rebeló. Y por eso me llevó al Colegio Nacional de Inválidos Civiles [de Murcia], la antigua ANIC. El colegio nos vino muy bien a la mayoría, en la que yo me meto porque yo estaba muy sobreprotegida [...] Al principio no me gustaba nada. Claro, yo no quería orden porque en casa ya me lo daban todo ordenado. Pero estuvo muy bien porque nos permitió independizarnos. Allí era «si algo haces mal, lo has hecho tú, es tu responsabilidad». Vas haciendo tus amigos, te vas soltando. Sabes, como que soy yo. En mi infancia no tenía muchas amigas, porque tenía que estar quieta. Ahí aprendí por ejemplo que había que ducharse diariamente, cosa que en los pueblos entonces... no. Hay que ducharse, hay que tener ordenado el armario, hay que tener unas horas de estudio. Y todo con cariño (ED_5).

Como nuestra entrevistada nos señalaba, la estancia en el centro les permitió a aquellos niños y niñas mitigar la influencia de sus familias, aprender las obligaciones más básicas y la disciplina en el estudio, en la 
higiene, en la vida diaria, etc., fomentando así sus capacidades para la vida social. En el mismo sentido se expresa nuestra siguiente entrevistada, quien además introduce un elemento muy interesante y recurrente en las estancias en «instituciones de encierro ${ }^{39}$ —no solo colegiospara personas con discapacidad:

Yo fui al colegio desde el principio, desde que lo abrieron en el 71, aunque antes iba a otro. Allí estaba muy bien. Fíjate que mis padres se fueron a vivir a Lérida y me dejaron ahí, pero porque yo quería. Yo era allí la reina. Era de las mayores, y había gente peor que yo. Entonces llevaba un bastón, pero no aparato. Y había gente con silla de ruedas, aparatos, deformaciones. Así que yo era la reina, allí me sentía muy bien (ED_4).

Esta autopercepción individual como la «reina» situada en la cúspide de aquel grupo de niños tanto por edad como por "capacidades», es como decimos una imagen muy recurrente tanto entre nuestros entrevistados y entrevistadas como en otros estudios de este tipo. ${ }^{40}$ Se trata, en efecto, de «operaciones de jerarquización» entre los residentes capaces de establecer rangos bien definidos en relación con las habilidades de los internos: los que andan con dificultad, los que necesitan silla de ruedas, los que tienen dificultades para manejar una silla de ruedas manual, los que carecen de brazos o piernas, etc. De hecho, aquellas niñas que se sentían «las reinas del mambo» —una expresión muy repetida en los testimonios recogidos de las antiguas alumnas- eran las encargadas de asistir a las menores y las que se encontraban en peores condiciones físicas y/o motoras:

Yo tenía a mi cargo a algunas que estaban peor. Había una niña que se llamaba Rocío que andaba con las manos. Tenía las piernas operadas, llevaba un pañal, y andaba con las manos. [Había literas] y yo recuerdo que dormía en la litera de arriba, y abajo estaba esta niña, Rocío, y yo la ayudaba. Incluso fui madrina

\footnotetext{
${ }^{39}$ Michel Foucault, Vigilar y castigar. Nacimiento de la prisión (Madrid: Siglo XXI, 2005). Y Erving Goffman, Internados. Ensayos sobre la situación social de los enfermos mentales (Buenos Aires: Amorrortu, 2012). Estos trabajos, conviene recordarlo, fueron publicados en 1975 y 1961 respectivamente.

40 Tracy Odell, «Not your average childhood: lived experience of children with phsysical disabilities raised in Bloorview Hospital, Home and School from 1960 to 1989», Disability and Society, 26 (1) (2011): 49-63, https://doi.org/10.1080/09687599.2011.529666.
} 
de las niñas que hicieron allí la comunión, porque había también niños abandonados, que los habían abandonado sus padres, porque tenían alguna discapacidad. Era todo discapacidad física, casi todo de polio, todo físico (ED_4).

No obstante, no todos los alumnos terminaban acostumbrándose a la vida como internos. Así recordaba en este sentido una de nuestras entrevistadas el episodio de ansiedad que obligó a su traslado a casa en plena madrugada:

Mi madre me internó, los profesores la convencieron. Pero duré quince días porque yo soy de tipo nervioso. Por la noche, el olor a sopa en aquellos pasillos me sentía muy mal. Emocionalmente me vino fatal. De tal manera que una noche me tuvieron que llevar a mi casa porque tenía un ataque de nervios. Me sentía rechazada por mi familia. Mis hermanos estaban en mi casa y yo aquí internada: «¿Por qué? ¿Soy un bicho raro o qué?» (ED_3).

Aunque aquella niña permaneció en el colegio como alumna hasta el final de su escolarización primaria — no en régimen de internado-, es interesante aquí subrayar esa percepción de «bicho raro» a la que hace referencia en este fragmento. Con sus palabras describe de hecho ese proceso de labeling —etiquetamiento- al que Goffman y Becker ya se habían referido en los años sesenta del siglo XX, esa incorporación de subjetividades formalizada en establecimientos destinados supuestamente a la reinserción de «sujetos desviados». ${ }^{41}$ En efecto, aunque aquellos niños no se correspondían con los actores privilegiados de estos sociólogos —enfermos mentales, drogadictos, delincuentes, etc.-, estos procesos de etiquetaje afectaban igualmente a las personas portadoras de deficiencias físicas al ser internados en instituciones específicamente creadas para ellos. Aunque estos espacios eran ideados en principio para curar, readaptar y educar, en ellos se reforzaban las imágenes sociales en relación a la discapacidad imperantes en aquel momento, al tiempo que jugaban un papel crucial en la creación de subjetividades y en los procesos de etiquetaje y en la propia definición de «desviación». Así lo expresaba una de nuestras entrevistadas:

\footnotetext{
${ }^{41}$ Erving Goffman, Internados; y Howard Becker, Outsiders. Hacia una sociología de la desviación (Madrid: Siglo XXI, 2009).
} 
A la mayoría a los que les ha quedado secuela creo que es por eso. Se han quedado un poco traumatizados por aquel encierro. Te sientes un poco desplazado de la familia, porque tienes algo que no encaja. [Recuerdo] que salíamos todos de excursión por el pueblo y aquello a mí me acomplejaba mucho y me daba mucho corte. Cuando la gente nos miraba por la calle. Bajábamos del autobús y éramos como bichos raros que habían traído por compasión al cine del pueblo. Y claro, yo era del pueblo, y no quería que mis «amigas normales» [sonríe], entre comillas, me viesen con aquella gente tan rara. Aquello era como un submundo, estaba ahí porque tenía que estudiar. Pero cuando salía de ahí era otro mundo. Porque claro, los complejos a esa edad eran muy duros. Sin embargo, era muy feliz allí porque estaba entre mis iguales [...] Por aquel entonces salías a la calle y la gente te miraba, te miraba mucho porque eras eso, rara. En ese mundo, de puertas para adentro, «todos son como yo, andan como yo, y estamos bien» (ED_3).

Aunque este caso es especialmente dramático debido al hecho de que nuestra entrevistada había nacido en el pueblo donde se ubicaba el colegio, lo cierto es que estos procesos de labeling son también fácilmente detectables en los testimonios de los niños y niñas provenientes de otros lugares, e incluso entre los trabajadores del centro. En este sentido se expresa nuestro siguiente entrevistado, administrativo en el colegio de Churra, que narraba como fue precisamente su llegada al centro lo que le hizo comprender la magnitud de la cuestión de la discapacidad y las necesidades de las personas portadoras de deficiencias físicas:

Empiezo a trabajar en Churra [...] en el colegio de la ANIC. [Y] ahí es mi primer contacto con la discapacidad. Ahí de golpe me encuentro con 200 críos con discapacidad, con maestros con discapacidad, ahí todo el mundo tenía discapacidad. Fue un choque que lo tuve que asumir y asimilar. Porque no lo entendía. Yo no entendía qué hacer [con] un niño de 5 años, de 6 años, que era de Galicia o de Asturias, separado de sus padres, [internado] allí. Porque llevaba bitutor. No lo entendía. Ahí estaban toda la primaria hasta octavo. Y los maestros todos, los trabajadores, o con discapacidad o familiares de personas con discapacidad. La ANIC era eso: discapacidad (ED_7). 
Nuestro protagonista en este caso no había sido plenamente consciente de su discapacidad precisamente hasta que no comenzó a trabajar en el colegio de Churra, en parte porque su familia lo había tratado siempre como a un igual y además tenían una posición económica suficiente. Los recuerdos de nuestros entrevistados, por lo demás, nos permiten componer la imagen social de la discapacidad vigente en la sociedad española de aquellos años, y ello a pesar de que cada vez ésta se hacía más visible y de que las personas con discapacidad se estaban integrando paulatinamente en la sociedad. A grandes rasgos, las actitudes negativas hacia las «diferencias» que daban lugar a procesos de exclusión o marginación y autoexclusión parecía seguir sobreviviendo en aquellos años. Así lo volvemos a comprobar en el siguiente fragmento de entrevista:

Como éramos todos [discapacitados]... Es más, yo, como cojeaba poco, me creía la reina del mambo, y cuando salí de allí me pegué un golpe enorme, porque claro, cuando me abrí a la sociedad me di cuenta de que era también discapacitada. Hay momentos que se pasan mal, porque no he tenido amigos en el pueblo, siempre gente de fuera. Te has sentido acomplejada. [Recuerdo que] nos reunían en el salón de actos y nos decían: «Tenéis que ser conscientes de que sois cojos, y cuando salgáis tenéis que estar mentalizados [...] Ni os vais a casar, ni vais a tener críos, ni nada». Y claro, eso te hace pensar que no eres una persona normal, y cuando sales sí que te pegas un tortazo. Porque ahí éramos todos iguales (ED_2).

Vuelve a hacerse evidente aquí esa «jerarquía entre internos» a la que antes hicimos referencia, y lo reconfortante que podía suponer compartir el mismo espacio con otras personas con discapacidad. Aun así, no cabe duda de que uno de los objetivos de este tipo de instituciones era la construcción de sujetos sometidos y ejercitados, «cuerpos dóciles» que, aún en sus limitaciones, fueran capaces de aumentar sus capacidades en términos económicos de «utilidad». ${ }^{42}$ En este sentido, la propia organización espacial y temporal de la vida diaria de los niños allí internos puede tomarse como un ejemplo idiosincrásico del funcionamiento

${ }^{42}$ Michel Foucault, Vigilar y castigar: 139-174. 
de estos dispositivos disciplinarios y de las propias instituciones de encierro:

Estábamos internas. Por la mañana te levantabas [...] Las habitaciones eran un poco deprimentes si ahora lo pienso. Eran como un salón inmenso dividido, y en mi habitación había 6 literas. [...] Al fondo las duchas, había una monitora que trabajaba allí. Te llamaban, íbamos a las duchas, hacíamos fila, y a desayunar. Eran mesas de 4, y las que éramos mayores nos ponían a darles de comer [a las que peor estaban]. Después las clases. Por la tarde hacíamos algunos «sus labores», otros hacían pinturas, luego unas horas libres y la cena. Como un internado (ED_4).

Así como la prisión «creaba» la subjetividad del «delincuente», el hospital psiquiátrico al «enfermo mental» y el colegio al «escolar», estas nuevas instituciones pensadas para el internamiento y la educación de los niños portadores de deficiencias físicas inauguraban nuevas formas de subjetivación de las personas con discapacidad en la España contemporánea. ${ }^{43}$ De este modo, en el funcionamiento del colegio de Churra podemos ver el desarrollo y la aplicación de saberes expertos, técnicas y discursos que parecían conectar los nuevos modelos sociales, culturales y médicos sobre la discapacidad con los cuerpos y las mentes de los allí internos. Es curioso en este sentido que tan solo una de nuestras entrevistadas se refiriera a la rehabilitación «que teníamos un día o dos a la semana» (ED_4), un hecho que tan solo puede señalar —a nuestro juicio- lo habitual que era para aquellos niños y niñas el ser sometidos a las técnicas e instrumentos del modelo médico o rehabilitador de la discapacidad desde su más tierna infancia. En aquel colegio, los «aparatos», las operaciones y los períodos de rehabilitación eran elementos familiares en la vida de los colegiales, y de ahí que esta medicalización de la discapacidad no sea en absoluto relevante en sus recuerdos. Sí lo era, ya lo vimos, cuando abandonaban aquellas reconfortantes paredes que los «hacían iguales en sus diferencias» y tenían que enfrentarse a entornos «normalizados».

Estos centros aparecían, así, como la conformación más evidente de esa nueva conciencia sobre la discapacidad desplegada desde finales

${ }_{43}$ Cayuela y Martínez-Pérez, «El dispositivo de la discapacidad...». 
de los años cincuenta, emergida entre las distintas instancias estatales, pero también entre los colectivos de afectados y las distintas organizaciones sociales. Gobernando los cuerpos y las mentes de aquellos niños portadores de deficiencias físicas, el Estado concedía a través de estos centros un nuevo impulso a la institucionalización de la discapacidad, construyendo al tiempo una realidad social que parecía «naturalizar las diferencias». En este sentido, no cabe duda de que el Centro de Asistencia y Educación Especial de ANIC «Nuestra Señora de la Fuensanta» de Churra aumentó sin duda las posibilidades de «integración social» de los niños y las niñas allí educados, pero también contribuyó a institucionalizar los procesos de etiquetaje y estigmatización social aún hoy persistentes en nuestras sociedades.

\section{CONSIDERACIONES FINALES}

Como hemos podido comprobar en nuestro caso de estudio, la creación de centros educativos por parte de la ANIC desde finales de los años sesenta puede sin duda ser considerada un auténtico hito en la formación de las personas con discapacidad física en la España contemporánea. A pesar de las limitaciones económicas a las que nuestro centro tuvo que hacer frente, los testimonios de nuestros entrevistados y entrevistadas parecen certificar el éxito de los programas de educación especial impulsados por la ANIC en las postrimerías del régimen franquista. En este sentido, los niveles educativos del colegio de Churra parecían por lo general destacables, en un contexto en el que la vocación universalista promulgada por la LGE no hacía sino despegar. Además, en el colegio de Churra nuestros entrevistados y entrevistadas consiguieron una formación más que suficiente que a la postre les iba a permitir bien acceder a empleos adecuados a sus capacidades y aspiraciones, o bien continuar en algunos casos con estudios superiores. De hecho, todos ellos lograron - a veces con mayor esfuerzo, pero sin grandes problemas según sus testimonios- desarrollar una carrera profesional como administrativos, decoradoras de interiores, técnicos informáticos o modistas. Al tiempo, su paso por aquel centro les supuso ampliar su «espacio social», multiplicando sus relaciones personales y adquiriendo hábitos y destrezas que contribuyeron a la postre a que alcanzaran una vida independiente y autónoma de una parte, y a desembarazarse de un medio social y familiar en ocasiones excesivamente sobreprotector de 
otra. En este sentido, de hecho, prácticamente todos los alumnos con los que hemos conversado se han casado y han tenido hijos.

No obstante, y por otro lado, la constitución de estos centros de educación especial como lugares de «marginación educativa» pudo contribuir precisamente a ahondar en la estigmatización y diferenciación social de las personas portadoras de deficiencias físicas en nuestro país. Como hemos analizado, estos centros funcionaron en ocasiones como auténticas «instituciones de encierro» donde ciertas formas de normalización reproducían el «modelo de la prisión» analizado por Foucault, produciendo una cierta «subjetividad del discapacitado» difícilmente amoldable después a los ambientes normalizados. Al tiempo, los procesos de labeling o etiquetaje, tan recurrentes en las instituciones creadas con el objetivo de corregir o normalizar las «desviaciones», son más que evidentes entre los recuerdos de aquellos niños y niñas. En este sentido, y aunque por supuesto el coste emocional de esa "condición de inválido» era más evidente para los colegiales que venían de lugares próximos a estos centros, el peso de esa «identidad desviada» se ha hecho evidente en mayor o menor medida en todos los testimonios de nuestros entrevistados y entrevistadas.

Sea como fuere, lo cierto es que estos centros educativos - junto con el Instituto Guttmann de Barcelona creado en 1965 y sus centros de formación profesional y centros de empleo protegido- fueron la realización más completa de la ANIC, así como su canto de cisne. Como también hemos señalado, las nuevas posibilidades abiertas por su inclusión en los Planes de Desarrollo II y III le permitió afrontar proyectos de mayor alcance y duración, pero ese impulso pareció apagarse ya antes incluso de la llegada de la democracia. La excesiva burocratización de la asociación, las luchas intestinas entre los distintos ministerios y las propias «familias» del régimen implicadas en el gobierno de la discapacidad, además de las crecientes demandas de cambio y exigencias provenientes de un movimiento asociativo cada vez más convencido de sus derechos y posibilidades ${ }^{44}$, certificaron la obsolescencia de una entidad muy alejada ya de las necesidades del colectivo. Como ya vimos, el recién

\footnotetext{
44 Mercedes Del Cura González y José Martínez-Pérez. «From resignation to non-conformism: association movement, family and intellectual disability in Franco's Spain (1957-1975)». Asclepio. Revista de Historia de la Medicina y de la Ciencia 70, no. 2 (2018): 232-244, https://doi.org/10.3989/ asclepio.2016.21; y Emilia Martos, «Activismo, movimientos y participación social de las personas
} 
creado SEREM pronto tuvo que hacerse cargo del centro de Churra - y de sus centros hermanos-, certificando su cierre poco después. Por lo demás, las nuevas estrategias en educación especial parecían certificar lo caduco del modelo impulsado por la ANIC, al tiempo que lo hacían los propios procesos de desinstitucionalización de la discapacidad progresivamente adoptados ya desde finales de los años setenta. Con todo, sería incorrecto no reconocer cómo este modelo, aún con sus carencias y limitaciones, permitió a muchos de nuestros entrevistados y entrevistadas adquirir una formación que, a la postre, sería fundamental en su desarrollo tanto profesional como personal.

\section{Notas sobre los autores}

Salvador Cayuela Sánchez es doctor en Filosofía por la Universidad de Murcia (2010) y doctor en Antropología Social por la Universidad Rovira i Virgili de Tarragona (2015). Ha sido profesor en las universidades de Castilla-La Mancha, Copenhague y Basilea, y actualmente es profesor en la Facultad de Filosofía de la Universidad de Murcia (España). Sus líneas de investigación principales han sido, entre otras, la biopolítica de la España franquista y de otros regímenes fascistas del siglo XX, la historia de la medicina, la antropología filosófica o la antropología de la salud. Entre sus obras escritas podemos destacar Por la grandeza de la patria. La biopolítica en la España de Franco (1939-1975), 2014; la coedición (junto a Antonio Campillo y Carmen Guillén) del libro Las (in)seguridades de Europa: una perspectiva crítica, 2017; o entre sus numerosos artículos «From walls to society. Institutions and the government of disability in contemporary Spain», en la revista Disability and Society, 37 (7), 2020.

Mercedes del Cura González es licenciada en Filosofía y Letras por la Universidad Autónoma de Madrid y doctora por la Universidad de Castilla-La Mancha. Profesora Titular de Historia de la Ciencia en las Facultades de Medicina y Farmacia de Albacete (UCLM) y miembro del Grupo de Estudios Sociales de la Discapacidad del Instituto de Investigación

con diversidad funcional durante la transición a la democracia», Historia contemporánea, 58 (2018): 747-779, https://doi.org/10.1387/hc.18128. 
en Discapacidades Neurológicas (UCLM). Ha sido investigadora visitante en el CSIC y en la Universidad de Swansea (UK). Ha desarrollado su investigación en el ámbito de la historia social de la medicina, la psiquiatría y la discapacidad, prestando especial atención a las problemáticas médico-sociales de la infancia y de las personas con discapacidad intelectual en la España del siglo XX. Entre sus trabajos se incluyen: Medicina y Pedagogía: la construcción de la categoría «infancia anormal» en España (1900-1939) (CSIC, 2011); Subnormality under debate: discourses and policies on intellectual disability during the late Franco regime. História, Ciências, Saúde. Manguinhos (2016); Bolstering the greatness of the Homeland: Productivity, Disability and Medicine in Franco's Spain (1940-1966), Social History of Medicine (2015); y Childhood, disability and vocational training in Franco's Spain during the 1950s and early 1960s, History of Education Review (2021) (los dos últimos en coautoría con José Martínez Pérez).

\section{REFERENCIAS}

Ballester Añón, Rosa. «Los organismos sanitarios internacionales y la rehabilitación de los niños con discapacidades físicas (1948-1975)». Revista de Estudios do Seculo XX 12 (2012): 89-101, http://dx.doi.org/10.14195/16478622_12_5.

Ballester Añón, Rosa. «España y la Organización Mundial de la Salud. La cuestión española y la puesta en marcha de políticas y programas de salud pública (1948-1970)» en Salud, enfermedad y medicina en el franquismo, coordinado por María Isabel Porras; Lourdes Mariño y María Victoria Caballero, 43-56. Madrid: Catarata, 2019.

Becker, Howard. Outsiders. Hacia una sociología de la desviación. Madrid: Siglo XXI, 2009.

Ben-Moshe, Liat; Chris Chapman and Alison Carey (eds.). Disability Incarcerated: Imprisonment and Disability in the United States and Canada. New York: Palgrave Macmillan, 2014.

Brégain, Gildas. «Nous ne demandons pas la charité. Nous voulons du travail!». La politique franquiste d'assistance aux invalides». Alter. European Journal of Disability Research 7 (2013): 206-221, https://doi.org/10.1016/j.alter.2013. 04.003. Brégain, Gildas. «Los debates públicos para sustituir el calificativo de inválido. (Argentina y España, de 1930 a 1970)», en Alter-habilitas: Percezione della disabilità nei popoli, editado por Silvia Carraro, 65-87. Verona: Alteritas, 2018. 
Brydan, David. Franco's Internationalist: social experts and Spain's search for legitimacy. Oxford: Oxford University Press, 2019.

Cayuela Sánchez, Salvador y José Martínez-Pérez. «El dispositivo de la discapacidad en la España del tardofranquismo (1959-1975): una propuesta de análisis». Asclepio. Revista de Historia de la Medicina y de la Ciencia 70, n. 2 (2018): 232-244, https://doi.org/10.3989/asclepio.2018.16.

Del Cura González, Mercedes. «El Servicio Social de Recuperación y Rehabilitación de Minusválidos: de la valoración de capacidades a la atención integral (1970-1979)», en Salud, enfermedad y medicina en el franquismo, coordinado por María Isabel Porras; Lourdes Mariño y María Victoria Caballero, 262-280. Madrid: Catarata, 2019.

Del Cura González, Mercedes y José Martínez-Pérez. «From resignation to non-conformism: association movement, family and intellectual disability in Franco's Spain (1957-1975) ». Asclepio. Revista de Historia de la Medicina $y$ de la Ciencia 70, n. ${ }^{\circ} 2$ (2018): 232-244, https://doi.org/10.3989/asclepio.20 16.21.

Del Cura González, Mercedes y Salvador Cayuela Sánchez. «De la caridad a los derechos: la discapacidad en el franquismo y la transición democrática», en El estado del bienestar: entre el franquismo y la transición, editado por Damián González y Manuel Ortiz, 259-288. Madrid: Silex, 2020.

Delgado Gómez-Escalonilla, Lorenzo. «International Organizations and educational Change in Spain during the 1960s». Encounters in Theory and History of Education 21 (2020): 70-91, https://doi.org/10.24908/encounters.v21i0.14334.

Foucault, Michel. Vigilar y castigar. Nacimiento de la prisión. Madrid: Siglo XXI, 2005 [Surveiller et punir. Naissance de la prison. Paris: Gallimard, 1975].

Goffman, Erving. Internados. Ensayos sobre la situación social de los enfermos mentales. Buenos Aires: Amorrortu, 2012 [Asylums. Essays on the Social Situation of Mental Patients and Other Inmates. New York: Doubleday, 1961].

González, María del Mar. «La legislación educativa y los alumnos con discapacidad: necesidad de actualización». Anuario Facultad de Derecho-Universidad de Alcalá 5 (2012): 81-105, http://hdl.handle.net/10017/13441.

Hudson, Bob. «Deinstitutionalization what went wrong? ». Disability, Handicap and Society 6, n. ${ }^{\circ} 1$ (1991):21-36, https://doi.org/10.1080/02674649166780021.

Jiménez Lara, Antonio y Agustín Huete García. «Políticas públicas sobre discapacidad en España. Hacia una perspectiva basada en los derechos». Política y sociedad 47, n.o 1 (2010): 137-152.

Lehmann, Albrecht. "Cultural Anthropology and Narratology», en Anthropological Perspectives. Tools for the Analysis of European Societies/Perspectivas antropológicas. Materiales para el análisis de las sociedades europeas, editado por Klaus Schriewer y Salvador Cayuela Sánchez, 69-91. Murcia/Münster: Waxmann/Editum, 2014. 
Martínez-Pérez, José. «La Organización Científica del Trabajo y las estrategias médicas de seguridad laboral en España (1922-1936)». Dynamis. 14 (1994): 131-158.

Martínez-Pérez, José. «Work, Disability and Social Control: Occupational medicine and Political Intervention in Franco's Spain (1938-1965)», Disability Studies Quarterly 37, nº 4 (2017), http://dx.doi.org/10.18061/dsq.v37i4.6098.

Martínez Pérez, José y Mercedes Del Cura González. «Bolstering the greatness of the homeland: productivity, disability and medicine in Franco's Spain (1938-1966) », Social History of Medicine 28, no. 4 (2015): 805-824, https:// doi.org/10.1093/shm/hkv060.

Martos, Emilia. Personas mayores y diversidad funcional física e intelectual durante la transición a la democracia. Problemática, reivindicación y actuación en el ámbito nacional y local, el ejemplo de la provincia de Almería. Almería: Universidad de Almería, Tesis Doctoral, 2014.

Martos, Emilia. «Trabajo y “minusvalía” durante el primer Franquismo: La asociación nacional de inválidos civiles», en La Historia: lost in translation?, editado por Damián A. Gonzalez y Manuel Ortiz Pérez, 2787-2796. Albacete: Ediciones de la Universidad de Castilla-La Mancha, 2017.

Martos, Emilia. «Activismo, movimientos y participación social de las personas con diversidad funcional durante la transición a la democracia». Historia contemporánea 58 (2018): 747-779, https://doi.org/10.1387/hc.18128.

Odell, Tracy. «Not your average childhood: lived experience of children with physical disabilities raised in Bloorview Hospital, Home and School from 1960 to 1989 ». Disability and Society 26, n . 1 (2011): 49-63, https://doi.org/ 10.1080/09687599.2011.529666.

Puelles Benítez, Manuel de. Educación e ideología en la España contemporánea. Madrid: Tecnos, 1999.

Richardson, Pamela K. «The School as Social Context: Social Interaction Patterns of Children with Physical Disabilities». The American Journal of Occupational Theory 56, no. 3 (2002): 296-304, https://doi.org/10.5014/ajot.56.3.296.

Schriewer, Klaus y Manuel Nicolás Meseguer. «El relato de justificación. Una herramienta para el análisis del franquismo». Revista Murciana de Antropología, 23 (2016): 85-102, https://revistas.um.es/rmu/article/view/273781.

Spivakovsky, Clare; Kate Seear and Adrian Carter (eds.), Critical perspectives on coercive interventions: law, medicine and society. London: Routledge. 2018. 